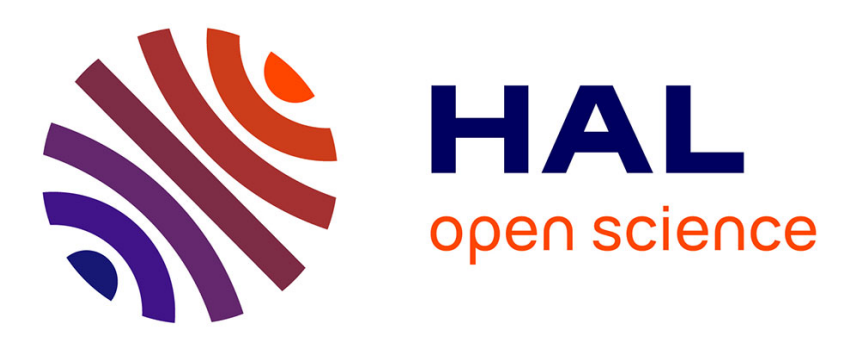

\title{
Le personnel politique catalan et aragonnais d'Alphonse Ier en Provence (1166-1196)
}

Martin Aurell

\section{To cite this version:}

Martin Aurell. Le personnel politique catalan et aragonnais d'Alphonse Ier en Provence (1166-1196). Annales du Midi : revue archéologique, historique et philologique de la France méridionale, 1981, 93 (152), pp.121-139. 10.3406/anami.1981.1953 . halshs-01305581

\section{HAL Id: halshs-01305581 https://shs.hal.science/halshs-01305581}

Submitted on 17 Jun 2016

HAL is a multi-disciplinary open access archive for the deposit and dissemination of scientific research documents, whether they are published or not. The documents may come from teaching and research institutions in France or abroad, or from public or private research centers.
L'archive ouverte pluridisciplinaire HAL, est destinée au dépôt et à la diffusion de documents scientifiques de niveau recherche, publiés ou non, émanant des établissements d'enseignement et de recherche français ou étrangers, des laboratoires publics ou privés. 


\section{Le personnel politique catalan et aragonais d'Alphonse ler en}

\section{Provence (1166-1196)}

Martí Aurell i Cardona

Citer ce document / Cite this document :

Aurell i Cardona Martí. Le personnel politique catalan et aragonais d'Alphonse ler en Provence (1166-1196). In: Annales du Midi : revue archéologique, historique et philologique de la France méridionale, Tome 93, N¹52, 1981. pp. 121-139;

doi : 10.3406/anami.1981.1953

http://www.persee.fr/doc/anami_0003-4398_1981_num_93_152_1953

Document généré le 13/03/2016 


\section{резюме}

Каталлонское и арагонское политическое окружение Альфонса 1-го в Провансе (1166-1196).

Статья изучает окружение Альфонса 1-го на основе декретов королевской канцелярии в Провансе. В эпоху несрвершеннолетия этого монарха гранды Ка- таллон/и и Арагона и высшее духовенство выполняют роль регентов. После 1176 г. их заменяют недав но разбогатевший рыцари и специалисты права. Вдобавок теже выходцы Пиринейского полуострова реорганизуют администрацию графства и ведут королевский контроль над восточной Превансой.

\section{Résumé}

L'article étudie l'entourage d'Alphonse ler à partir des actes provençaux de la chancellerie du roi. Pendant la minorité de celui-ci, les Grands catalans et aragonais ainsi que le Haut Clergé assurent la régence. Après 1176, ils sont remplacés par des chevaliers récemment enrichis et par des techniciens du Droit. En outre, ce sont des hommes venus de la Péninsule ibérique qui réorganisent l'administration du comté et assurent le contrôle royal sur la Provence orientale.

\section{Abstract}

The article studies the entourage of Alphonse I from the Provencal Acts of the King's chancellor's offices. While Alphonse was still a minor, the Catalan and Aragonese Grandees as well as the High Clergy assured the regency. After 1176, they were replaced by recently enriched knights and by legal technicians. Further, it was men from the Iberian peninsula who reorganized the County's administration and assured royal control over eastern Provence.

\section{Zusammenfassung}

Dieser Artikel untersucht die Umgebung von Alfons I. anhand der provenzalischen Akten der königlichen Kanzlei. Während seiner Minderjährigkeit führten die katalanischen und aragonischen Granden sowie der hohe Klerus die Regentschaft. Nach 1176 treten erst vor kurzem reich gewordene Ritter und rechtskundige Fachleute an ihre Settle. Außerdem wird die Verwaltung der Grafschaft von Leuten aus der iberischen Halbinsel neugeordnet, die dem König die Herrschaft über die östliche Provence sichern. 


\section{LE PERSONNEL POLITIQUE CATALAN ET ARAGONAIS $D^{\prime} A L P H O N S E I^{\text {er }}$ EN PROVENCE (1166-1196)* *}

Le chemin de l'approche sociologique du pouvoir d'un prince au Moyen âge a été frayé par l'historiographie française des dernières décennies, à la suite d'un bon nombre d'historiens du Droit qui, sous y'égide de J.-F. Lemarignier, rejoignaient les rangs de l'histoire sociale' ${ }^{1}$ Leur méthode - le dépouillement et l'interprétation des listes des témoins et des souscriptions des actes des instances supérieures - * permet de reconstituer le système d'une taxinomie sociale $\star^{2}$. Nous avons essayé de l'appliquer à l'étude des quelques cent vingt-cinq actes du pouvoir en Provence sous Alphonse $I^{\text {er }}$ (1162/66-1196), premier comte de Barcelone devenu roi d'Aragon. Nous ne présentons ici que les conclusions auxquelles nous sommes parvenu sur le corps politique d'origine catalane et aragonaise dans le comté. Il comprend deux groupes d'hommes dont le rôle est différent : d'une part, un certain nombre de chevaliers et de prélats qui accompagnaient habituellement le premier comte-roi dans ses itinéraires ibériques et ne font à sa suite que des séjours intermittents en Provence $^{3}$; de l'autre, les Catalans et les Aragonais qui séjournaient de façon stable dans le comté pour y représenter localement son pouvoir.

* 14, avenue Jules-Isaac, 13100 Aix-en-Provence.

** Le texte de cet article est extrait de quelques chapitres d'un mémoire de maitrise sur Le Pouvoir comtal en Provence sous Alphonse $I^{\circ 0}$, dirigé par $M$. Noël Coulet, que nous remercions vivement, et soutenu à l'Université de Provence en juin 1980.

1. LEMARIGNIER (J.-F.), Le gouvernement royal aux premiers temps capétiens (978-1108) (Paris, 1965); GuILlot (0.), Le comte d'Anjou et son entourage au XI' siècle (Paris, 1972); BoURNAZEL (E.), Le gouvernement capétien au XII' siecle (Limoges, 1975). Cf. GuENÉ (B.), Les tendances actuelles dans l'histoire politique du Moyen Age, dans 100 Congrès des Sociétés Savantes (Paris, 1977), p. 46 ss. et Gurensson (J.), Tendances, méthodes et techniques nouvelles de l'histoire médiévale, ibidem, pp. 8-13.

2. DUBY (G.), Les trois ordres ou l'imaginaire du féodalisme, Paris, 1978, p. 185.

3. Ce groupe présente d'autant plus d'intérét pour l'histoire de la Catalogne qu'il correspond au conseil permanent du roi, permettant d'ecarter les témoins dits occasionnels, présents dans les actes ibériques du roi en fonction de la proximité géographique de leurs domaines des lieux d'émission des actes royaux et de cerner ainsi le groupe des conseillers les plus proches du monarque. Sur les problèmes méthodologiques du témoignage dans les actes du pouvoir, cf. GurLor, op. cit., t. II, pp. 5-20. 


\section{L'entourage permanent d'Alphonse I*}

Les membres de l'entourage catalan du premier comte-roi peuvent être regroupés en quatre listes : la première comprend les Grands de la principauté ; la deuxième, les hauts prélats; la troisième est celle d'un ensemble de chevaliers de second rang, arrivés dans l'entourage d'Alphonse $\mathrm{I}^{\text {or }}$ à la fin de sa minorité ; la quatrième, celle des légistes, mettant leurs connaissances juridiques au service du monarque. Avec eux, quelques Aragonais accompagnaient le roi.

\section{Les Grands catalans}

Les membres des vieilles dynasties comtales ou vicomtales de Catalogne ne sont guère venus en Provence dans le cortège d'Alphonse I"r. Seule, la famille de Besalù fait exception. Hug de Bas (1145-1185), vicomte depuis 1154, demeura auprès du roi dans les Alpes et dans les cités du Bas-Rhône l'hiver 1176-1177. Fils de Pons de Cervera et d'Almodís, sœur de Raimond Bérenger le Saint (1131-1162), ce cousin germain du roi venait de participer à l'expédition contre Valence dans l'ost royal (1175). Néanmoins, son voyage de 1176 en Provence répondait moins au service du monarque qu'à une raison de politique familiale: la difficile installation en Sardaigne de sa sœur Agalbursa de Bas, épouse en secondes noces de Barison, juge d'Arborea. En août 1177, Hug figure parmi les témoins de l'accord qui partage les droits sur le monnayage d'Arles entre l'archevêque Raimon de Bollène (1163-1182) et le comte de Provence Raimond Bérenger IV (1168-1181), frère du roi. La même année, Hug de Bas s'embarquait pour l'île, où il mourait en 1185. C'est alors qu'Alphonse $I^{\circ r}$, à la suite des accords conclus avec les Génois à Calatayud et à Hyères, par l'intermédiaire du comte de Foix, alors procureur de Provence, envoya des hommes en Sardaigne qui vinrent renforcer Agalbursa et son neveu Hug Pons (1185-1221), fils d'Hug de Bas et d'une dame sarde, dans leur guerre contre Petro, fils du premier mariage de Barison, pour s'assurer le judicat d'Arborea. L'affaire finit par le partage de 1192, qui consacra l'installation définitive du lignage dans l'îlet. Pons de Cervera (1151-

4. Certains historiens, à la suite d'une malheureuse erreur d'ordre onomastique commise par BARTHELEMY (L.). Inventaire chronologique et analytique des chartes de la maison de Baux (Marseille, 1882, pp. 655 ss.) avaient cru voir la famille des Baux dans celle des nouveaux juges d'Arborea. Malgré la réfutation dont cette théorie avait fait l'objet par MrRET I SANS (J.). Los vescomtes de Bas en l'illa de Sardenya (Barcelone, 1901), passim, et par MARTIN-CHABOT (F.-E.), \&a politique hors d'Espagne d'Alphonse II, roi d'Aragon *, dans Position de Thèses de $l$ 'Ecole des Chartes, 1902, pp. 10-12, elle traîne encore dans les manuels : BARATIER (E.), Histoire de Provence (Toulouse, 1969, p. 139), et dans les monographies les plus récentes: SMYRL (E.), La famille des Baux (X-Xur siècles), dans Cahiers du C.E.S.M., $\mathrm{n}^{\circ} 2$ (Gap, 1968), p. 30. 
195), frère d'Hug et régent de la vicomté de Besalù à partir de 1185, aisait partie de l'entourage comtal lors de l'avènement du comte-roi in Provence (1166-1167). En 1189, il apportait son aide militaire au roi dans ses campagnes en Provence orientale contre Boniface de Castellane et contre la ville de Nices.

Bien que dépourvue d'un titre vicomtal, la famille de Montcada jouissait alors en Catalogne d'une puissance comparable aux Bas. Guillem Ramon de Montcada (1118-1173), « le grand sénéchal \#, est un des personnages les plus importants de la vie politique du XIr siècle catalan. En 1118, il accéda au dapiférat que son lignage détenait depuis le milieu du Xre siècle. En 1136, au retour de son exil en Aragon auprès de Ramire le Moine (1134-1137), il retrouvait son office dans la cour barcelonaise. Il joua un rôle capital dans la poussée catalane vers le Sud au détriment de l'Islam, qui caractérisa le règne de Raimond Bérenger le Saint. Il participa à l'expédition contre Almería (1147) et aux conquêtes de Tortosa (1148) et de Lleida (1149), cités de la Nouvelle Catalogne dans lesquelles Guillem Ramon se taillait d'importantes coseigneuries. Il encouragea la fondation du monastère cistercien de Santes Creus sur ses terres par des moines de Grandselve. Le comte de Barcelone l'avait institué son exécuteur testamentaire. Raimond Bérenger, qui se dirigeait avec son neveu le comte de Provence à Turin pour rendre hommage à l'empereur Frédéric Barberousse, trouva la mort en août 1162. Guillem Ramon assura donc la tutelle du jeune Alphonse $I^{\text {er }}$ avec Guillem de Torroja, l'évêque de Barcelone, prenant en main la direction des affaires catalanoaragonaises pendant sa minorité. A ce titre, il collabora, ainsi que son fils Guillem de Montcada (1152-1172), seigneur du Béarn à partir de 1170 , à la difficile installation du comte-roi en Provence, face à Raimond $\mathrm{V}$ de Toulouse $^{6}$. A la même époque, leur parent Adelbert

5. Mrquen I Roseul (F.), Liber Feudorum Maior, Barcelone, 1945-1947 (ci-dessous LFM), n' 892 (octobre 1176); Delaville LE RoulX (J.), Cartulaire général de l'ordre de Saint-Jean de Jérusalem, Paris, 18941906, $\mathrm{n}^{\circ} 502$ (octobre 1176); Arxiu de la Corona d'Aragó (ci-dessous ACA), Pergamins d'Alfons $I^{\circ}, \mathrm{n}^{\circ} 214$ (janvier 1177) ; Arch. dép. Bouches-du-Rhône (ci-dessous ADBR), 3 G 16, Livre Noir, fo $41 \mathbf{v}^{\circ}$ (août 1177) : après lui, dans la liste de témoins, apparaît Berenguer de Sardaigne Caruana (J.), Itinerario del rei Alfonso $I^{\circ}$ de Cataluña, II ${ }^{\circ}$ de Aragón, dans Estu dios de Edad Media de la Corona de Aragón, t. VII (1967), p. 159 (octobre 1186) Liber Jurium Reipublicae Genuensis, Turin, 1854-1857, t. I, $\mathbf{n}^{\circ} 341$ (8 octobre 1186) Pons I Maroues (J.), Cartulari de Poblet, Barcelone, 1938 (ci-dessous CP), $n^{\circ} 9$ (septembre 1167); ADBR, B 293 (octobre 1189), B 292 (octobre 1189); SOBREQUES VIDAL (S.), Els barons de Catalunya, Barcelone, 1957, pp. 41 ss.; Diccionari biogrd fic, Barcelone, 1966-1970, t. I, pp. 230-234.

6. LFM n $n^{\circ} 494$ (11 octobre 1162); ACA, Alfons $1^{\circ}, \mathrm{n}^{\circ} 42$ (avril 1167), $\mathrm{n}^{\circ} 47$ (octo bre 1167); CP, $\mathrm{n}^{\circ} 96$ (septembre 1167); D'AcHERI (L.), Spicilegium sive collecti veterum aliquot scriptorum qui in Galliae bibliothecis delituerant, Paris, 1723 t. III, pp. 539-540 (septembre 1167); BonNASsIE (P.), La Catalogne du milieu di $X \bullet d$ la fin du XII siècle: croissance et mutations d'une société, Toulouse, $197 \xi$ n. 710 : Diccionari.... t. III, pp. 237 ss. 
de Castellví souscrivait les diplômes provençaux du roi; ce lignage servait le comte de Barcelone au moins depuis le milieu du $\mathrm{x}^{\mathrm{e}}$ siecle et avait profité récemment de l'expansion catalane sur les terres sar. rasines; les Castellví devaient l'hommage au roi pour les places de Tivissa (Ribera de l'Ebre), de Mora et de Garcia (Priorat) ${ }^{7}$.

A la mort du grand sénéchal, son deuxième fils, Ramon de Montcada $(† 1188)$ hérita du tiers de Tortosa et de biens en Pallars, à Tarragone et à Lleida. Bien que le dapiférat lui revint théoriquement, il n'exerça jamais cet office, sans doute à cause d'une politique de vacance voulue par le roi à l'égard de cette trop puissante famille. Ramon se rendit encore à l'île de Jarnègues, entre Tarascon et Beaucaire, lors du premier traité de paix entre le roi et Raimond $V$ de Toulouse en avril 1176. Sa présence symbolisait peut-être la figure de son père disparu. Il ne resta que peu de temps auprès d'Alphonse $I^{\circ *}$ dans les cités rhodaniennes ${ }^{8}$. Les sources provençales perdent sa trace à partir du mois de mai, époque à laquelle il devait revenir dans ses domaines catalans. C'était la dernière fois qu'un Montcada faisait partie du conseil du premier comte-roi. Dès lors, ce clan devenu trop influent se trouvait écarté du pouvoir.

A tous les niveaux de la politique royale, les années autour de 1176 sont des années charnières. Elles correspondent à la fin de la minorité d'Alphonse Ir ${ }^{\circ}$. D'après $R$. d'Abadal, le comte-roi aurait alors pris en main le gouvernement de son royaume et signé la paix de Jarnègues pour pouvoir se consacrer pleinement à ses domaines d'outrePyrénées, délaissant le Midi de la France ${ }^{10}$. En outre, l'abandon, provisoire - il est vrai - du conflit avec le comte de Toulouse sanctionne un fait capital dans l'histoire provençale: à partir de 1178, les comtes de Provence quittent Tarascon et Arles et choisissent leur résidence principale à Aix, alors que leur centre d'action se déplace vers l'Est'11. De même, d'importants remaniements affectent à ce moment-là l'entourage comtal qui perd ouvertement son caractère de conseil de régence. L'effacement du haut clergé catalan est fort significatif de ces mutations.

7. Arch. dép. Vaucluse, G 15, fo 113 vo-114 (13 avril 1166) ; ADBR, 3 G 16, fo 35 (mars 1167); ACA, Alfons $I^{\circ}, n^{\circ} 42$; LFM n 794 (17 août 1167); CP $\mathrm{n}^{\circ} 96 ;$ LFM $\mathrm{n}^{\circ} 240$ (décembre 1174); BonNASSIE, op. cit., p. 286.

8. LFM, n० 899 (18 avril 1176); CP, $n^{\circ} 178$ (21 avril 1176) ; LFM, n 894 (26 avril 1176) ; Udina MaRToRel (F.), El \& Llibre blanc de Santes Creus $*$, Barcelone, 1947 (ci-dessous LBSC), $\mathrm{n}^{\circ}$ 191, avril 1176); ADBR, $6 \mathrm{G} 438$, Livre jaune, $\mathrm{f}^{\circ} 7$ avril 1176).

9. Il serait né en mars 1154 (Soldevila (F.), História de Catalunya, Barcelone, 1063, 2 éd., p. 193, note 97) ou en mars 1157 (UBIETo (A.), De nuevo sobre el nacimiento de Alfonso II de Aragón, dans Estudios de Edad Media de la Corona de Aragón, t. VI (1956), pp. 203-209).

10. * A propos de la domination de la maison comtale de Barcelone sur le Midi français $\%$, dans Annales du Midi, 1964, p. 336.

11. COULET (N.), Aix-en-Provence, espace et relations d'une capitale (milieu $X I V^{*}$ siecle-milieu $\left.X V^{*}\right)$, thèse ronéotypée, Aix, 1979, pp. 2-12. 


\section{Les prélats}

Un grand nombre de prélats catalans, que nous énumérons par la suite, s'étaient rendus entre Rhône et Durance en 1166 pour assurer la succession du premier comte-roi. Ils attirèrent alors le haut clergé provençal dans le camp du comte de Barcelone, faisant ainsi échec aux prétentions de Raimond $V^{12}$. Guillem de Torroja (1144-1175), évêque de Barcelone, puis archevêque de Tarragone, était le pilier du conseil de

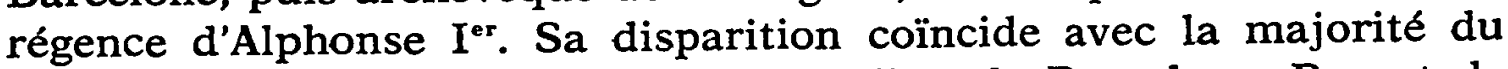
premier comte-roi. Son successeur au siège de Barcelone, Bernat de Berga (1172-1189) participa à la campagne provençale de 1176. Hug de Cervelló, archevêque de Tarragone (1163-1171), était issu d'une vieille famille du comté d'Ausone qui avait acquis, autour de l'an mil, des biens dans la zone frontalière entre le Llobregat et le Gaià. Il assista, en tant que sacriste de la cathédrale de Barcelone, à la rédaction du testament de Raimond Bérenger le Saint. Devenu archevêque de Tarragone, il fut mêlé aux luttes pour la domination de la ville, face aux descendants du normand Robert Bordet, conquérant de la cité, et trouva la mort au cours d'une vendetta le 17 avril 1171. Guillem de Peratallada, évêque de Gérone (1161-1168), est le constructeur du noyau de l'ancien palais épiscopal de la ville du Ter. L'évêque de Vic, Pere de Redorta (1146-1185) fut le procureur de Raimond Bérenger IV en Provence en $1173^{13}$. Mais, après 1176, ils n'échappèrent point à la laïcisation de l'entourage royal. Une nouvelle génération prenait alors leur relève.

\section{Les nouveaux venus}

C'est le moment, en effet, où s'affirme le rôle de la famille de Vilademuls. Sous Raimond Bérenger le Saint, Ramon de Vilademuls faisait partie du conseil comtal dès 1143 ; en 1157, il était comitor judex. Puis, pendant la minorité d'Alphonse $I^{\bullet r}$, sa lignée disparut de la scène politique catalane pour y revenir de plein droit à la fin. De 1176 à 1181, Arnal de Vilademuls, sans doute le fils de Ramon, conseilla assidûment le roi durant ses voyages provençaux. Ramon de Vilademuls, son frère, devint viguier du comte dans le Roussillon. Il apposa son signum sur quelques actes provençaux de 1176 à 1191. Un

12. L'archevêque d'Arles cède les châteaux de Fos et d'Albaron à Alphonse $\mathbf{I}^{\text {er }}$ : Albanes (J.), Chevaliter (U.), Gallia Christiana Novissima, Montbéliard-Paris, 18991920 (ci-dessous GCNN), Arles, $\mathrm{n}^{\circ}=615,616,617$ (mars 1167). A l'époque, les prélats provençaux fréquentent assidûment l'entourage royal.

13. GCNN, $\mathrm{n}^{\circ}$ 615; ADBR, B 286, B 31, f० 239 (8 mai 1167); LFM, n॰ 794 ; CP, ne 96 ; D'ACHERI, loc. cit.; MORIS (H.), BLANC (E.), Cartulaire de l'abbaye de Lérins, Paris, 1883-1905 (ci-dessous CL), t. II, $\mathrm{n}^{\circ} 8$ (1168); DoUBlet (G.), Recueil des actes concernant les évêques d'Antibes, Monaco-Paris, 1915 (ci-dessous AEA), no 85 (1166) et $n^{\circ} 89$ (juillet 1173) ; SOLDEVILA, op. cit., p. 200 ; BoNNASSIE, op. cit., pp. 287 291 ; Diccionari..., t. I, pp. 553-554 et t. III, p. 445. 
troisième membre de la famille, Berenguer de Vilademuls, abbé de Sant Feliu de Gérone, puis archevêque de Tarragone (1175-1194), fréquenta régulièrement la curia regis. En avril 1176, il se trouvait en Provence auprès du roi. L'année suivante, Berenguer participait à la campagne de Cuenca et s'occupait activement de la création d'un bon nombre de villes et de la mise en valeur de la Nouvelle Catalogne : les cités de Mont-Roig (qu'il fonda en 1180), de Vilagrassa (1185) et de Vilafranca (1191) en fournissant des exemples. Sa carrière fut interrompue en 1194. Il fut assassiné à Matabous par Guillem Ramon de Montcada $(+1224)$, fils de Marie de Béarn, lors de la révolte des grands pyrénćens contre le roi et l'évêque d'Urgell'14.

Nous retrouvons de même à partir de 1176 le signum de Guillem d'Alcarràs dans les chartes provençales : ce chevalier participa alors activement à la campagne du comte en Provence Orientale. Il fut, notamment, son fidéjusseur à l'égard de la ville de Nice ${ }^{15}$. Il était le fils de Gerai de Jorba (1146-1188) : conseiller de Raimond Bérenger le Saint, spécialement à partir de 1150 , il avait jadis coopéré à la prise de Lleida (1149). A la mort du comte de Barcelone, il figura parmi les personnages qui confirmèrent son testament à Huesca à la demande de la reine Pétronille. Geral s'était rendu en Provence dans la suite du comte-roi enfant en $1167^{16}$. Le domaine de la famille s'étendait principalement dans la Nouvelle Catalogne ${ }^{17}$. Geral et sa femme Saurina avaient cédé des terres pour la constitution du temporel du monastère cistercien de Santes Creus. Le 10 février 1176, le roi et la reine Sancia inféodaient les castra de Montblanch et de la Riba, dans la vallée du Francolí, à Geral de Jorba et à son fils Guillem d'Alcarràs. Le testament de Guillem montre l'état de sa fortune: les disponibilités en espèces sont très élevées. En effet, son père Geral de Jorba avait été le délégué en Catalogne du roi Loup de Murcie et de Valence, Ibn Mardanix, dont il tenait à ferme le tribut qu'il devait au comte de Barcelone. Les dirhems arabes et les terres obtenues par

14. LFM, $\mathrm{n}^{\circ} 899$; ADBR, $6 \mathrm{G} 438$, f 7 ; Bouche (H.), Chorographie et Histoire de Provence, Aix, 1664, t. II, p. 148 (23 juin 1176); AEA, $\mathrm{n}^{\circ} 92$ (juin 1176); AEA, $\mathrm{n}^{\circ} 91$ (juin 1176); ADBR, B 287 (juin 1176); LFM, $\mathrm{n}^{\circ} 892$; ACA, Alfons $\mathrm{I}^{\circ}, \mathrm{n}^{\circ} 214$ (Janvier 1177) ; GUERARD (B.), Cartulaire de Saint-Victor de Marseille, Paris, 1857 (ci-dessous CSV), $\mathrm{n}^{\circ} 902$ (21 mai 1177), $\mathrm{n}^{\circ} 1110$ (mai 1177), $\mathrm{n}^{\circ} 759$ (3 juin 1177), $n^{\circ} 903$ (27 janvier 1178); ADBR, B 289 (décembre 1178); Bouche, op. cit., t. II, p. 152 (23 octobre 1179); AEA, n० 104 (juin 1181); LFM, $n^{\circ} 900$ (février 1185); ADBR, B 292 (octobre 1189), 5 G 91, fo 99 ; LFM, n' 253 (29 arvil 1157) ; Diccionari..., t. IV, pp. $460-461$.

15. AEA, $\mathrm{n}^{\circ} 92, \mathrm{n}^{\circ} 91, \mathrm{n}^{\circ} 90$ (1176); LFM, $\mathrm{n}^{\circ} 893$ (juin 1176); CSV, $\mathrm{n}^{\circ} 902, \mathrm{n}^{\circ} 1110$, n' 759 .

16. D'ACHERI, loc. cit.; LFM, n॰ 494 (11 octobre 1162).

17. Jorba: province de Barcelone, partit judicial d'Igualada. Alcarràs : province et partit judicial de Lleida. 
la faveur royale dans la Nouvelle Catalogne rendent compte de la fortune de ces parvenus ${ }^{18}$.

Guillem de Claramunt (1160-1194) était aussi un des fidèles les plus zélés du roi et s'était rendu à plusieurs reprises en Provence dans sa suite $^{10}$. Il avait participé aux dernières campagnes pour refouler les Sarrasins au-delà de l'Ebre; en 1153, Guillem se trouvait au siège de Siurana. En 1160, il faisait donation à Santes Creus de ses biens au Codony, près de Tarragone. En 1188, il donnait la permission au monastère de Sant Cugat de construire des moulins à Igualada. Un autre facteur explique, au même titre que le service du comte, sa réussite sociale: les liens de clientèle, sous la forme de relations de vassalité et de parenté que Guillem entretenait avec la famille vicomtale de Cardona ${ }^{20}$. Un autre conseiller du roi, Guillem d'Anglesola, qui vint en Provence dans la décennie 1180-1190, avait, comme lui, bénéficié de la poussée catalane vers le Sud: il avait ajouté à son domaine familial dans le comté de Pallars, des terres conquises sur les musulmans que le roi lui inféoda en $1188^{21}$.

Ainsi, parallèlement à l'éviction ou à la désertion des grands laïcs et ecclésiastiques de l'entourage comtal, un groupe d'hommes nouveaux accédait aux centres de décision. Leur statut social reflète l'aspect ministériel de la suite d'Alphonse $I^{*}$.

\section{Les légistes}

En outre, le comte-roi devait s'appuyer, pour mener à bien la grande réorganisation administrative et institutionnelle qui caractérise fondamentalement son règne ${ }^{22}$, sur un groupe de juristes, dont les connaissances allaient apporter les assises techniques et idéologiques à l'extension de son pouvoir.

Pere de Cardona ( $t$ avant 1185) est un bon exemple de ces juristes au service du comte. Ce chanoine de Vic, sacriste de la cathédrale de Barcelone, doctor legum magnificus ${ }^{23}$, accompagna, avec son parent

18. LBSC, $\mathrm{n}^{\circ} 146$; ACA, Alfons $\mathrm{I}^{\circ}, \mathrm{n}^{\circ} 192$ et $\mathrm{n}^{\circ} 212$; Diccionari..., t. II, pp. 484 485.

19. LFM , n० 899 ; LBSC, $\mathrm{n}^{\circ} 191$ (avril 1176) ; CSV, $\mathrm{n}^{\circ} 902, \mathrm{n}^{\circ} 1110, \mathrm{n}^{\circ} 759$; GCNN, Marseille, $\mathrm{n}^{\circ} 182$; Bibliothèque Méjanes d'Aix, fonds de la Celle, $\mathrm{n}^{\circ} 347$; ACA, Alfons $I^{\circ}, \mathrm{n}^{\circ} 364$ (mars 1183 ).

20. Diccionari... t. I, p. 573; Hec est convenencia que est facta inter Guillelmus de Cardona vicecomitem et Geraldam vicecomitissam uxorem suam et Guillelmus de Claramonte, consanguineum et hominem eorum, ACA, Alfons $\mathrm{I}^{\circ}, \mathrm{n}^{\circ} 542$.

21. AEA, $\mathrm{n}^{\circ} 105$ (août 1181); ADBR, $5 \mathrm{G} 91$, fo 99 . Il est le vassal du roi pour le château de Mur (Conca de Tremp), LFM, $n^{\circ} 169$ (novembre 1192) et $n^{\circ} 621$ (6 septembre 1188).

22. VentuRA (J.), Alfons el Cast (Barcelone, 1961), p. 230 ; Busouet (R.), Le Moyen Age in Encyclopédie des Bouches-du-Rhône (Marseille, 1923), t. II, p. 532.

23. Nécrologe de Vic, cité par Valls-TaBerneR (F.), Le juriste catalan Pierre de Cardona, cardinal de l'église romaine sous Alexandre III, dans Mélanges Paul Fournier, Paris, 1929, p. 746. 
Berenguer de Cardona, Alphonse $I^{\text {er }}$ en Provence, dans son voyage de 1176 à 1177. En 1179, Pere demeurait encore dans le comté, où il assistait avec Raimond Bérenger IV à une donation à l'église de Fréjus $^{24}$. Il se rendit ensuite à Montpellier, auprès de Placentin, le célèbre juriste bolonnais : là, il traduisit en latin des constitutions grecques. En 1182, il était promu cardinal au titre de Saint-Laurent in Damaso $^{25}$. Il appartenait sans doute à la maison vicomtale d'Ausone, mais sa situation exacte dans la généalogie de la famille n'a pu être établie $^{28}$. Son état clérical nous fait plutôt penser à un cadet de la tige vicomtale ou à un membre d'une de ses branches collatérales, qui avait conservé son rang social grâce à ses connaissances techniques et au service du monarque.

La fortune de la famille de Bernat de Caldes était d'origine bien plus récente. Bernat, notaire royal au moins depuis 1167 , était le frère de Ramon de Caldes (1135-1200), doyen de Barcelone et compilateur du Liber Feudorum Maior ${ }^{27}$. Bernat de Caldes dressa un grand nombre d'actes provençaux entre 1167 et $1177^{28}$. En 1171 , il avait rejoint son frère Ramon au sein du chapitre de Barcelone et devenait également chanoine de Lleida, ville dans laquelle Bernat et Ramon acquéraient quelques maisons. Leur père, Porcell, avait jadis été le baile du comte de Barcelone pour ses domaines autour de Caldes de Montbui (Vallès Oriental); leur frère aîné, Porcellet, avait hérité du patrimoine paternel. C'est dans leur milieu familial et dans le chapitre de Barcelone que Bernat et Ramon de Caldes auraient acquis une formation administrative et notariale ${ }^{29}$. Dans la seconde moitié du $\mathrm{XII}^{\mathrm{e}}$ siècle, en effet, les chapitres méridionaux constituent une pépinière de légistes. Les instances supérieures savent, de leur côté, utiliser leurs services, comme le montrent les chanoines de Saint-Ruf, de Notre-Dame de Doms et de Saint-Trophime dans le Bas Rhône ${ }^{30}$. L'exemple de Pere de Cardona et de son séjour dans l'école de Montpellier est révélateur des rapports culturels qui pouvaient exister

24. LFM, $n^{\circ} 892$ (octobre 1176); ACA, Alfons $I^{\circ}, n^{\circ} 214 ;$ CSV, $n^{\circ} 903$; AEA, n' 98.

25. Gouron (A.), Autour de Placentin à Montpellier: Maître Gui et Pierre de Cardona, dans Studia Gratiana, 1976, pp. 349-350.

26. VALLS-TABERNER, art. cit., p. 744.

27. Cf. le remarquable article de Bisson (Th. N.), Ramon of Caldes (c. 1135c. 1200) : Dean of Barcelona and King's Minister, dans Law, Church and Society: Essays in Honor of Stephan Kuttner, Pensylvanie, 1977, pp. 281-292.

28. Bouche, op. cit., t. II, p. 147; LFM, $n^{\circ} 794$; ACA, Alfons I० n $^{\circ} 139$; LFM,

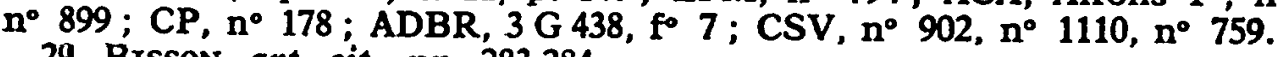

29. BISSON, art. cit., pp. 283-284.

30. Poly (J.-P.), Les légistes provençaux et la diffusion du Droit romain dans le Midi, dans Mélanges Aubenas, Montpellier, 1974, p. 628. 
entre l'ancienne Septimanie et la Catalogne pendant la période de la renaissance du droit romain ${ }^{31}$.

Deux autres notaires, Pons d'Osor et Guillem de Bassia, ont accompagné le roi à plusieurs reprises en Provences2. Ils siégeaient avec Bernat et Ramon de Caldes au Computum, le conseil devant lequel les bailes du roi rendaient compte de leur administration en Catalogne ${ }^{33}$. Pons d'Osor rendit justice avec le roi et avec le juge mage de Provence, Guillem Bernad d'Aix, lors d'un plaid dans le Var. Guillem de Bassia exerçait de même des fonctions judiciaires en Catalogne ${ }^{34}$. A l'époque, les notarii des comtes de Barcelone sont déjà les chefs d'une petite chancellerie. Ils se font aider de scribes pour la rédaction des chartes: le notaire Pere de Blandes qui se trouvait en 1184 dans la cour aixoise de Sanche, frère d'Alphonse $I^{\text {er }}$ et comte de Provence (1181-1185), passant ensuite au service du roi, avait le scribe Bernad Ucaes sous ses ordres ${ }^{35}$. Leur niveau social et leur fortune correspondaient a leurs attributions : en 1171, Pons d'Osor donnait le mansum d'Almafar au monastère de Sant Cugat; en 1195, Guillem de Bassia faisait donation au même établissement des terres qu'il avait achetées à Guillem de la Granada ${ }^{36}$.

Nous pouvons ainsi aisément conclure sur un certain nombre de traits spécifiques à l'entourage catalan et permanent d'Alphonse $I^{\circ r}$. Tout d'abord, l'éviction des grands - ni les comtes d'Urgell, de Pallars, d'Ampuries ni les vicomtes d'Ausone, de Barcelone ou de Gérone ne traversèrent le Rhône avec le roi -, ne nous étonne guère de la cour d'un prince de la fin du XIr siècle. Un nouveau corps politique s'est mis en place en Catalogne, au moins depuis le règne de Raimond

31. Gaudemet (J.), Le Droit romain dans la pratique chez les docteurs aux $\mathbf{X I}^{\mathrm{e}}$ et $\mathbf{X I I}^{\circ}$ siècles, dans Cahiers de Civitisation Médiévale, 1965, pp. 365-380; GouroN (A.), Diffusion des consulats méridionaux et expansion du droit romain aux XIF et XIII* siècles, dans Bibliothèque de l'Ecole des Chartes, 1963, pp. 26-76; Les ètapes de la pénétration du Droit romain au XII ${ }^{e}$ siècle dans l'ancienne Septimanie, dans Annales du Midi, 1957, pp. 103-120; Gropdanengo (G.). Vocabulaire romanisant et réalité féodale en Provence, dans Provence Historique, 1975, pp. 255-273.

32. Pons d'Osor: Bibliothèque Méjanes, fonds de la Celle, $n^{\circ} 347$ (16 novembre 1182); AEA, $\mathrm{n}^{\circ} 108$ (novembre 1182) ; AMDR, B 2, f० 41 (décembre 1182) ; LFM, $n^{\circ} 900 ; \mathrm{CL}, \mathrm{n}^{\circ} 10$; ADBR, B 293 (décembre 1189 et février 1190), B 294 (mars 1190), $B$ 1065, $\mathrm{f}^{\circ} 183 \mathrm{v}^{\circ}$ (mai 1193); Guillem de Bassia: LBSC, $\mathrm{n}^{\circ} 191$; ADBR, 6 G 438, $f^{\circ} 7$; Delaville Le RoulX, op. cit., $\mathrm{n}^{\circ} 502$; ADBR, B 289 ; AEA, $\mathrm{n}^{\circ} 103$ (mai 1181), $n^{\circ} 104$ (juin 1191); ACA, Alfons $\mathrm{I}^{\circ}, \mathrm{n}^{\circ} 364$ (mars 1183); GuICHARD (F.), Essai historique sur le cominalat de la ville de Digne, Digne, 1846, PJ, $\mathrm{n}^{\circ} 9$ (avril 1191).

33. Aragó CabaNas (A. M.), La institución * baiulus regis * en Cataluña en la época de Alfonso el Casto, dans VII ${ }^{\circ}$ Congreso de la Corona de Aragón, Barcelone, 1962, t. III, p. 139.

34. ADBR, 5 G91, fo 99 (février 1190); ACA, Alfons $I^{\circ}, n^{\circ} 456$ (juin 1187).

35. AEA, $n^{\circ} 111$; ADBR, 5 G 91, f० 99, B 292, B 293.

36. RIUS SERRA (J.), Cartulario de "Sant Cugat * del Vallés, Barcelone, 19451947 (ci-dessous CSC), $n^{\circ} 1076$ (8 août 1171) et $n^{\circ} 1206$ (5 septembre 1195). 
Bérenger le Grand (1082-1131); ces hommes étaient recrutés dans les * couches moyennes et subalternes de l'aristocratie, dans des familles qui par conséquent doivent tout à la faveur comtale ${ }^{37}$. Ils sont les descendants d'un groupe de ministériaux, enrichis à l'ombre des comtes de Barcelone, à la faveur de l'expansion de la Principauté vers le sud. La carte de leurs domaines, situés dans la Nouvelle Catalogne, est révélatrice d'un succès et d'une ascension sociale récents.

Un seul conseiller du roi en Provence provient d'une couche sociale plus élevée. Il s'agit de Pons de Mataplana (1122-1184) qui se rendit en Provence en 1176 et, sans doute, en 1181. Ses séjours dans le comté sont, en outre, attestés par des sources poétiques, les sirventès que Guillem de Berguedà écrivit à son sujet ${ }^{38}$. C'était un puissant seigneur, appelé marquès par le troubadour. Son château était situé dans le Ripollès, au cœur des Pyrénées catalanes. Il avait aussi des biens en Cerdagne. Pourtant, son genre de vie était proche de celui des chevaliers qu'il fréquentait dans l'entourage royal; il était adoubé et participa au tournoi de Sentfores (Vic). Pons de Mataplana est donc un bel exemple de l'intégration de la vieille noblesse a la chevalerie, du nivellement, au sommet de la hiérarchie sociale, entre les milites et les nobiles ${ }^{39}$. Son ennemi, Guillem de Berguedà, révolté avec les barons pyrénéens contre le comte de Barcelone et contre l'évêque d'Urgell dans les années $90 \mathrm{du}$ XIr siècle, était le porte-parole des insurgés de la vieille noblesse catalane qui voyaient leurs prérogatives diminuer face à la main-mise progressive du monarque sur leur ban. Guillem qualifia Pons de Mataplana de mendic, épithète fort significative du mépris que le troubadour éprouvait à l'égard de ce traître à la cause de son groupe social, celui des grands feudataircs de la Vieille Catalogne, en lutte contre Alphonse $I^{{ }^{\circ} 40}$. Sa présence, dans un entourage où la ministérialité prime, ne s'explique que par ce contexte politique.

$\mathrm{Si}$, à cette époque, le pouvoir du comte de Barcelone, unique et indiscutable seigneur de la Principauté, se renforce considérablement et si Alphonse Ir peut, non seulement se passer de l'aide des grands feudataires pour gouverner mais venir à bout de leurs insurrections, le monarque ne peut pas, en revanche, se dispenser de la collaboration d'un groupe d'experts en lois, de techniciens du droit - de technocrates, si l'on nous permet l'anachronisme - , chargés de la direc-

37. Bonnassie, op. cit., p. 710.

38. $\mathrm{LFM}, \mathrm{n}^{\circ} 893$; AEA, $\mathrm{n}^{\circ} 104$ (Montpellier, juin 1181); * Que mals augurs ac part Berra / quan se frais la gamb'esquerra (trad.: " il n'eut pas de chance du côté de Berre / quand il se cassa la jambe gauche $»$ ), poème $\mathbf{n}^{\circ}$ XI de la magistrale édition de RIQuer (M. de), Guillem de Bergueda, Poblet, 1971.

39. Contamine (Ph.) (sous la direction de), La Noblesse au Moyen Age, pp. 21-31.
40. Riouer, op. cit., t. I, pp. 76-100 et pp. 125-145. 
tion de sa chancellerie et dont les atttributions ne cessent de s'accrốtre. A travers ce dernier ensemble, nous entrevoyons ce que la cour deviendra un jour, lors de l'édification du véritable Etat Féodal du Bas Moyen Age.

\section{Les Aragonais}

Les Aragonais de l'entourage permanent d'Alphonse I ${ }^{\text {er }}$ paraissent, d'après les sources provençales, avoir été moins nombreux que les Catalans. Nous les retrouvons d'abord dans l'entourage de la minorité du roi, sans doute sous l'influence de la reine mère Pétronille, dont le plus proche collaborateur, Eximinio d'Artosilla, alférez alors, puis mayordomo du royaume d'Aragon, seigneur des honores de Bolea et de Loarre ${ }^{41}$, séjourna en Provence de 1166 à 1167. D'autres magnats accompagnaient le roi à cette occasion : Dodo de Alcalá, témoin de la donation que la reine avait faite du royaume d'Aragon à Alphonse $I^{\bullet r}$ le 18 juin 1164 ; Petro de Castellazuelo, alférez et seigneur de Calatayud; Eximinio Romeu, nommé seigneur de Tarazona par le roi; Petro de San Vicente, alférez en 1175, et, enfin, Garcia de Pola ${ }^{42}$.

Dix ans plus tard, Alphonse $I^{* r}$ revenait dans le comté. Parmi les témoins de la paix de Jarnègues nous retrouvons Blasco Romeu, mayordomo de la cour et seigneur de la tenencia de Saragosse ; Blasco de Maza $(+1182)$, seigneur de Borja; Artal de Alagón, qui deviendra par la suite seigneur d'Alcañiz, de Pedrola et de Borja et Petro de Tarroja, évêque de Saragosse (1153-1184), le seul ecclésiastique aragonais qui vint en Provence avec Alphonse $I^{\text {er }}{ }^{* 3}$.

A quelques exceptions près, les Aragonais disparaissent de la suite du roi en Provence jusqu'en 1190 ; mais, à partir de cette date, une nouvelle génération se met en place. Enneco de Aveo, seigneur de Ricla et d'Uncastillo, et Petro Cornel, mayordomo d'Aragon sous Jacques I* (1213-1276), étaient fidéjusseurs et otages pour le roi, dans le traité de juin 1193, par lequel Alphonse $I^{\text {er }}$ et les Baux prévoyaient la conquête et le partage de Marseille. La même année, Guillermo de Alcalá et Eximinio de Rada prêtaient serment au nom du roi dans l'accord conclu avec la ville d'Arles et un autre Aragonais, Garcia de Torres, est témoin

41. LFM, $\mathrm{n}^{\circ} 16$; LFM, $\mathrm{n}^{\circ} 17$; CP, n० 166 ; Ventura, op. cit., p. 92 ; Caruana (J.), Los alféreces de Aragón en tiempos de Alfonso II y Pedro II (1162-1213), dans Revista de Archivos, Bibliotecas y Museos, 1955, p. 413 (l'alférez dirige l'ost royal) ; Caruana (J.), Los mayordomos de Aragón en los siglos XII y XIII, ibid., 1956, pp. 365-366 (le mayordomo est le conseiller intime du roi, chargé de sa protection et du ravitaillement de la cour); sur la détention des seigneuries dans le royaume : LACARRA (J.M.), * Honores * et * Tenencias * en Aragón, au Xre siècle, dans Annales du Midi, 1968, pp. 485-527.

42. ACA, Alfons $I^{\circ}, n^{\circ} 47$; BOUCHe, op. cit., t. II, p. 147 ; LFM, n 794 ; D’ACHERI, loc. cit.

43. LFM, $\mathrm{n}^{\circ} 899$ (18 avril 1176). 
de l'acte 4 . D'autre part, des chevaliers catalans commencent alors à fréquenter l'entourage royal : c'est le cas de Bernard de Portella (1189. 1236), de Dalmau de Palou, de Pere de Creixell, de Guillem de la Gra. nada ou de Guillem de Cervera $(† 1245)^{45}$. Ces Aragonais et ces Catalans jeunes à l'époque, renouvelaient le personnel politique de la fin du règne du premier comte-roi ; quelques-uns allaient jouer un rôle de premier plan sous Pierre II (1196-1213) et sous Jacques $I^{* *}$. Ils appartiennent, en effet, à la génération de Muret. Mais, bien avant la fin du rêve occitan, tous ces chevaliers avaient franchi les Pyrénées avec le monarque pour rentrer dans leurs domaines. Sous Alphonse $I^{\text {or }}$, d'autres nobles originaires de la Péninsule Ibérique demeuraient en Provence; quelques-uns allaient y faire souche.

\section{Catalans et Aragonais installés en Provence}

Les Catalans et les Aragonais établis dans le comté devaient jouer un rôle essentiel dans le renforcement du pouvoir du prince en Provence. Certains d'entre eux occupèrent des charges plus ou moins importantes dans le gouvernement ou dans l'administration locale du comté. D'autres, ayant reçu des fiefs entre Rhône et Durance, fréquentèrent assidûment la curia comtale de 1166 à 1196.

\section{Les agents du comte}

Le règne d'Alphonse $I^{\text {or }}$ en Provence se caractérise par l'apparition de nouvelles institutions dans le gouvernement du comté. Les textes nous font alors découvrir les procureurs de Provence, les juges mages, les bailes locaux... Ces personnages étaient, dans leur grande majorité, d'origine ibérique.

Mis à part Barral ( $\uparrow 1192)$, vicomte de Marseille, aucun Provençal ne devait être titulaire de la baillie du comté, une charge qui faisait de ceux qui l'exerçaient de véritables vice-rois de Provence. Guillem de Bell-Lloc était procureur du roi dans le comté en 1171, auprès de Raimond Bérenger IV, alors enfant. En juin 1173, il était remplacé par l'évêque de Vic, Pere de Redorta. A cette époque, il est de retour à Barcelone, où il souscrit un acte de donation du roi à l'église de Santa Eulàlia et à la chapelle palatine ${ }^{46}$.

44. ADBR, B 296; ACA, Alfons I०, n० 538 (janvier 1190); ADBR, B 294, B 1065, I0 $183 \mathrm{v}^{\circ}$ (mai 1193), B 295 (juin 1193), B 294 (juin 1193); CL, $\mathrm{n}^{\circ} 11$.

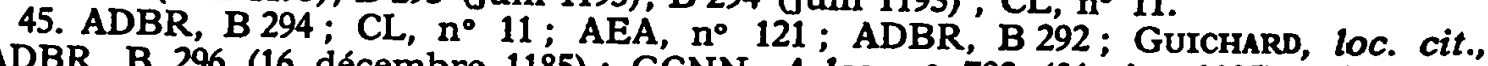
ADBR, B 296 (16 décembre 1185); GCNN, Arles, n' 703 (février 1195); D'ACHERI, Spicilegium..., t. III, p. 556 (1.r mars 1195); ADBR, 5 G 91, $\mathrm{f}^{\circ} 99$. 46. LFM, $n^{\circ} 483$ (juillet 1168, en Catalogne); CSV, $n^{\circ} 1109$ (1171); ACA, Alfons $I^{\circ}$,
$n^{\circ} 135$. 
Depuis le premier tiers du XIre siècle, la famille de Bell-Lloc avait été très liée à la cour comtale barcelonaise. A partir de 1133, Bernat de Bell-Lloc était devenu, avec son frère Pere Bertran de Bell-Lloc, le conseiller le plus assidu de Raimond Bérenger le Saint. Ils avaient, à ce titre, participé à plusieurs campagnes en Provence dans l'ost comtal ${ }^{47}$. Le noyau initial du patrimoine du groupe familial se situe dans la ville même de Barcelone, où les Bell-Lloc étaient les vassaux du vicomte Berenger de la Guardia pour quelques maisons, et à la Roca del Vallès, où ils détenaient le château. Comme les autres ministériaux du comte de Barcelone de la première moitié du XIr ${ }^{e}$ siècle, ils avaient arrondi leur patrimoine dans la Nouvelle Catalogne; là, les Bell-Lloc possédaient des terres à Cervera, à Cabra (Alt Camp) et à Aldea (Ribera de Segre $)^{48}$.

Guillem de Bell-Lloc était le fils de Pere Bertran et de Sancia. Son frère Ramon était chanoine du chapitre de Barcelone. Guillem était fort probablement le châtelain de la Roca. Pourtant, à son retour de Provence, dans les années 1180 , nous le retrouvons parmi les notables de la région de Cervera. Par là, son statut social rejoint celui des membres de l'entourage permanent d'Alphonse $I^{\text {er49 }}$.

Lope Eximinio fait partie de ce groupe d'Aragonais qui avaient envahi l'entourage du comte-roi à partir de 1190 . Nous le retrouvons pour la première fois dans la suite royale en mai 1191, prenant un rôle actif dans la conclusion du traité d'alliance contracté avec le roi de Portugal, à Huesca, et dirigé contre Alphonse VIII de Castille. L'année suivante, ce chevalier aragonais occupait la charge de procureur de Provence, auprès du jeune comte Alphonse II (1189-1209), fils du roi. En 1194, il quittait la Provence dans le cortège d'Alphonse I'r. Il devint, par la suite, l'un des chevaliers les plus fidèles de Pierre II et de Jacques I $I^{\text {erso }}$.

Deux aragonais, les frères Assalito de Gudal et Guillermo de Arbanés, le remplacèrent à la tête du comté. Assalito fréquentait l'entourage d'Alphonse $I^{\circ r}$ au moins depuis 1187, époque à laquelle il est témoin d'un acte royal dressé à Saragosse. Au début de l'année 1190, il

47. CSV, n॰ 822 (15 février 1134) ; ADBR, B 394 (11 juillet 1150), 3 H 6 (23 juillet 1150); CSV, n $^{\circ} 966$ (17 août 1150); ADBR, B 18, f० 69 (septembre 1150), B 279 (septembre 1150); GCNN, Marseille, $\mathrm{n}^{\circ} 148$ (sejtembre 1150); ADBR, B 281 (mai 1151) ; B 284 (1156) ; CSV, n 996 (12 août 1157).

48. LFM, $\mathrm{n}^{\circ} 347$; LBSC, $\mathrm{n}^{\circ} 222$ et 259 ; LFM, $\mathbf{n}^{\circ} 468$.

49. CSC, $n^{\circ} 996$ (1154); LBSC, $n^{\circ} 259$ (13 mai 1183), n 269 (2 février 1185), n० 387 (20 juin 1196) - MAs (J.), Notes historiques del Bisbat de Barcelone, 13 vol., Bar. celone, 1906-1921) : vol. XI-XIII, Libri Antiquitatum (ci-dessous LA), n' 1566 (1॰r mars 1144), $\mathrm{n}^{\circ} 1568$ (16 mars 1144), $\mathrm{n}^{\circ} 1572$ (4 mai 1144), $\mathrm{n}^{\circ} 1609$ (18 juillet 1146), $n^{\circ} 1857$ (14 mars 1163), $n^{\circ} 1915$ (11 avril 1168).

50. Caruana, « Itinerario... » p. 266 (mai 1191); CSV, no 978 (1192-1193) ; ADBR B 1065, $\mathrm{f}^{\circ} 183 \mathrm{v}^{\circ} ; \mathrm{CL}, \mathrm{n}^{\circ} 11 ; \mathrm{CP}, \mathrm{n}^{\circ} 267$ (février 1194); Bernat Desclot, Crònica. éd. Soldevila, Barcelone, 1971, p. 608. 
accompagna le roi en Provence et dans la Péninsule Ibérique. A la mort du premier comte-roi il devint l'un des principaux conseiller: de Pierre II, qui le nomma seigneur d'Alcázar. Ce légiste fut l'envoy، de Jacques I* au roi André II de Hongrie pour conclure le mariage du roi d'Aragon avec sa fille Violante (1235). Il était, sans doute, appa renté à Petro de Arbanés, conseiller d'Alphonse II en Provence jus qu'à la mort du comte en 1209, date à laquelle il regagna la cour de Pierre II en Catalogne ${ }^{51}$.

Garcia de Resa était de même très probablement aragonais, établi en Provence autour de 1176. L'année suivante il faisait partie des pro. ceres curie de Raimond Bérenger IV. C'est en 1179 qu'il souscrivit un acte comtal en tant que juge mage de Provence, puis Garcia se rendit au siège de Fourques avec le roi. Il devait être par la suite nommé viguier d'Albaron, castrum dont on connaît l'importance stratégique. Il apparaît pour la dernière fois dans les textes en 1194, en tant que fidéjusseur d'Alphonse $I^{e r}$ dans son accord avec les consuls a'Arles $^{52}$. Le fils de Garcia de Resa, Guillem de Cotignac, reçut son fief patronymique des mains d'Alphonse II au début du xrrre siècle. II devint baile en 1222, sous Raimond Bérenger V (1209-1245) ${ }^{53}$. Les familles de ces Aragonais casés dans le comté continuaient de fournir le personnel politique nécessaire aux comtes de Provence de la maison de Barcelone.

La carrière de Iohan de Artasona présente une certaine ressemblance avec celle de Garcia de Resa. Le 16 novembre 1182, il est témoin à Hyères d'une donation du roi au monastère de la Celle, mais ce n'est qu'à partir de 1190 qu'il fréquente la cour comtale d'une façon assidue. En 1195, alors que la ville acquiert le rôle de "capitale * administrative du comté, il devient baile d'Aix. Il quitte cette charge en 1206 pour occuper, ultérieurement, celle de procureur de Provence ${ }^{54}$.

$\mathrm{Si}$ le poids des Catalans et des Aragonais se fait sentir dans les institutions centrales, il en va de même sur le plan local. D'autant plus

51. ACA, Alfons $\mathrm{I}^{\circ}, \mathrm{n}^{\circ} 538$; ADBR, B 38 fo 122 et f० $117 \mathrm{v}^{\circ}$; ADBR, B 295 ; ADBR, B 296 ; GCNN, Arles, no 703 ; D'ACHERI, op. cit., t. III, p. 556; CARUANA, *Itinerario... », p. 294 (13 août 1194); ACA, Alfons $I^{\circ}, \mathrm{n}^{\circ} 454$ (1187); LFM, n० 87 et $\mathrm{n}^{\circ} 226\left(5\right.$ avril 1198); LFM, $\mathrm{n}^{\circ} 416$ (6 juin 1199); Cronica de Jaume $\mathrm{I}^{\circ}$, éd. Soldevila, pp. 15 et 205 ; ARDISSON (C.), Etude sur l'entourage des comtes de Provence appartenant à la maisor: de Barcelone (1196-1226), Mémoire de D.E.S. dac-
tylographié (Aix, 1967), p. 18.

52. AEA, n ${ }^{\circ}$;0; ADBR, 3 G 16 fo $41 \mathrm{v}^{\circ}$; Bouche, op. cit, t. II, p. 152: AEA, $\mathrm{t}^{\circ} 103$; DEvIC (C.), VAISSETTE (J.), Histoire générale du Languedoc (Toulouse, 1875),

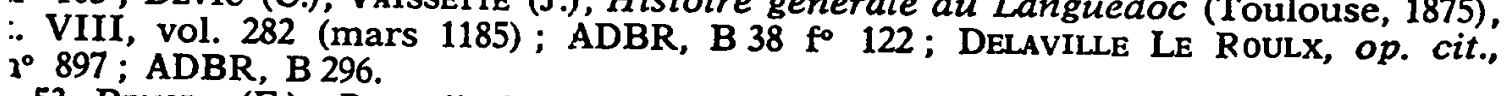

53. BENOIT (F.), Recueil des actes des comtes de Provence appartenant a la naison de Barcelone (1196-1245), Monaco-Paris, 1925, $\mathrm{n}^{\circ} 169$ (13-I-1233); CORTEZ ,F.), Les grands officiers royaux de Provence au Moyen âge, Aix ,1921, pp. 13 et 25. 54. Bibliothèque Méjanes, fonds de la Celle, no 347 ; ADBR, B 293; CSV, n 978 ;
IDBR, B 1065, fo $183 v^{\circ}$, B 295 ; D'ACHERI, op. cit., t. III, p. 556. 
que, dans la seconde moitié du XIre siècle, la Provence connaît des transformations profondes avec l'augmentation du nombre et l'élargissement des attributions des bailes des domaines comtaux. Ceux-ci cessent d'être les modestes intendants domestiques des périodes précédentes pour devenir des officiers ordinaires de police domaniale, des juges, des agents fiscaux, qui élargissent leur rayon d'action bien au-delà des limites strictes de la seigneurie comtale. Ils deviennent, somme toute, les véritables représentants du pouvoir comtal à l'échelon local ${ }^{5 s}$. Malheureusement, les textes, bien qu'ils nous permettent d'entrevoir cette évolution, passent trop souvent sous silence les noms des bailes domaniaux des comtes. Outre Garcia de Resa et Iohan d'Artasona, nous n'en connaissons que cinq. Trois d'entre eux sont, avec certitude, d'origine catalane ou aragonaise ${ }^{58}$.

En 1178, Pons de Brugueres prêtait serment aux vicomtes de Marseille au nom de Raimond Bérenger IV, s'engageant à respecter leur exemption fiscale: il occupait probablement une charge d'administration locale dans la région marseillaise. Preuve en est son rôle comme fidéjusseur et otage du comte dans le pacte passé avec les Baux pour la prise de la ville phocéenne. En outre, il est appelé bajulus dans l'acte par lequel Alphonse $I^{\text {er }}$ concédait la forêt d'Albaron aux Cisterciens ${ }^{57}$.

Ramon de Perella appartenait à une famille de petits châtelains possessionnés à Caldes de Malavella (Selva). En avril 1176, il garantissait par serment le respect des gages du roi que les Porcellets détenaient à Arles. Peut-être y occupait-il une fonction de baile du domaine royal. En novembre 1179, il est à Perpignan, apposant son signum dans l'eschatocole d'un parchemin royal après Arnal de Palou, un autre Catalan établi en Provence. Il était sans doute rentré dès cette date dans son domaine nord-catalan, d'où il rayonnait vers le Roussillon, récemment annexé au domaine du comte de Barcelone (1172) ${ }^{58}$.

Le nom du baile d'Eyguières, Gomiz, est fort probablement d'origine aragonaise. Ce personnage est témoin de l'immunité accordée par le roi à plusieurs églises provençales du chapitre de Saint-Ruf d'Avignon en février $1190^{50}$.

Ainsi, l'étude des listes des officiers et des bailes de Provence sous Alphonse $I^{* r}$ montre l'origine presque exclusivement catalane ou aragonaise de leurs titulaires. A tous les niveaux de l'administration, à

55. BUSQUET, op. cit., pp. 528-530.

56. Les deux autres bailes connus sont Guillem Raimon Gantelme, à Tarascon, et Pons Niel, à Aix (1176).

57. Archives municipales de Marseille, AA 2, $n^{\circ} 3$; ADBR, B 295 ; ADBR, B 294 ; $D^{\prime} A$ CHERI, op. cit., t. III, p. 556 ; ACA, Alfons $I^{\circ}, 712$ (décembre 1195).

58. LBSC, $n^{\circ} 191 ; A D B R, B 288 ; A E A, n^{\circ} 103 ; L F M, n^{\circ} 400$ (30 novembre 1183). 59. ADBR, B 293. 
l'échelon central ou local, la majorité des postes de gouvernement du comté étaient accaparés par des étrangers.

Membres occasionnels de la Curia comitis

D'autres nobles d'outre-Pyrénées fréquentaient alors la cour des comtes de Provence, sans y occuper, à notre connaissance, une charge précise. Avec ses vingt présences dans les actes des comtes de Provence jusqu'en 1183, Arnal de Palou en fournit un bon exemple. Sa jeunesse nous est bien connue grâce aux Libri Antiquitatum de la cathédrale de Barcelone. Il était le fils de Dalmau de Palou et de Stephania. Vers 1155, il était entré dans le chapitre de Barcelone, quelques années après son cousin Berenguer de Palou avec lequel il partageait sa mense capitulaire à Sant Julià de Palou, reçue en dot de leurs parents. Esprit inquiet, Arnal avait abandonné « l'état clérical pour suivre les voies du siècle ${ }^{60}$; pourtant, il y revint à Pâques 1161 , et fut réadmis dans le chapitre par l'évêque et par les chanoines. Repentance des plus précaires : au bout de quelques mois, il réglait ses affaires de succession avec son cousin, puis, en octobre 1162, vendait ses biens à l'église de Barcelone pour 100 morabatins. Arnal de Palou quittait aussitôt la Catalogne ${ }^{61}$. Il était passé alors au service de Raimond Bérenger III, comte de Provence, qui lui avait concédé des terres dans le diocèse de Vence. Le prince voulait sans doute profiter du bagage de culture juridique de l'ex-chanoine de Barcelone. En octobre 1165, il assista à Beaucaire à la conclusion du pacte contracté entre ce comte et Raimond $\mathrm{V}$ de Toulouse, au sujet du mariage de leurs successeurs. C'est cet accord qui, en prévoyant l'union du fils du comte de Toulouse avec la fille de Raimond Bérenger III, allait donner un fondement juridique aux prétentions de Raimond $\mathrm{V}$

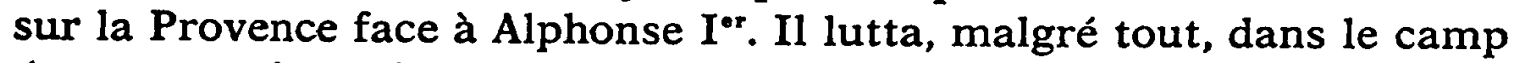
du comte-roi pendant les années 1166 et 1167 . En 1168, il rentrait en Catalogne avec le monarque. De retour en Provence, il était présent à la première paix de Jarnègues et séjourna à Tarascon et à Arles auprès du roi. En été 1176 , il participait à la campagne du roi en Provence Orientale. A partir de cette période et jusqu'en 1183, il demeura dans le comté, prêtant conseil à Raimond Bérenger IV et à Sanche, à l'exception de quelques intervalles et notamment pendant les voyages qu'il fit avec Alphonse $I^{\text {er }}$ en Roussillon au cours des années 1179 et 1180 . Nous retrouvons sa trace en Catalogne en 1188 ; là, un Arnal de Palou est témoin d'un acte royal à Anglesola. Nous

60. LA, $\mathrm{n}^{\circ} 1829$ (6 avril 1161).

61. LFM, $\mathrm{n}^{\circ} 804$ (19 novembre 1159) ; LA, $\mathrm{n}^{\circ} 1730$ (22 mai 1155), $\mathrm{n}^{\circ} 1757$ (24 avril 1157), $n^{\circ} 1767$ (1*r juin 1157), $n^{\circ} 1842$ (24 octobre 1161), $n^{\circ} 1851$ (4 octobre 1162). 
savons, par la vente que son fils, Joufré Baston, fit au roi de ses possessions provençales, qu'il était décédé avant octobre $1189^{\circ 2}$.

Ramon de Vilaseca, un autre Catalan installé dans le comté, était le fils de Pere de Vilaseca ( $†$ avant 1189), qui figure parmi les témoins d'une exemption accordée par Raimond Bérenger III au monastère cistercien de Silvacane en 1165. Pere avait reçu le castrum de la Roque-d'Anthéron, non loin de cette abbaye, des mains d'Alphonse $I^{e r}$. En decembre 1189, le roi en confirmait la possession à Ramon de Vilaseca, en échange de 2500 sous melgoriens. La forteresse lui était remise conformément au droit féodal catalan, qui fait de la reddition des châteaux une des principales obligations vassaliques ${ }^{63}$. Cette localité, dont l'importance stratégique dans la vallée de la Durance, zone frontalière du comté de Forcalquier, était capitale, se trouvait ainsi dans des mains sûres.

Rodrico de Cascante fréquenta les cours comtales de Raimond Bérenger IV, puis de Sanche. En janvier 1177, Alphonse $I^{\circ r}$ et son frère, le comte de Provence lui donnaient Vilhosc, près de Sisteron. En mai 1181, au cours du siège de Fourques, place défendue par le comte de Toulouse, auquel Rodrico avait apporté l'aide militaire au roi, il recevait le castrum du Chaffaut dans le canton de Digne ${ }^{64}$. L'emplacement de ces deux localités, dans cette région alpine hostile, où les comtes de Provence avaient besoin d'appuis solides, comme le prouvent la révolte de Boniface de Castellane en 1189, celle d'Uc de Montréal en 1190 et les méfaits de Blacasset en 1194, est fort significatif ${ }^{65}$. La lignée de Rodrico de Cascante devait se perpétuer dans les Alpes du sud au-delà du XIII ${ }^{e}$ siècle.

L'Aragonais Marchisio de Saragosse participa en juin 1176 au jugement rendu par Alphonse $I^{\text {or }}$ et par ses frères en faveur de l'évêque d'Antibes à Grasse. En mai 1181, il apportait son auxilium au roi dans

62. Poly (J.-P.), La Provence et la société féodale (879-1166), Paris, 1976, p. 323, note 47 ; ADBR, 3 H 6 (1165); LFM, $n^{\circ} 898 ;$ CSC, n० 1130 ; Arch. dép. Vaucluse, G 15, fo $113 \mathrm{v}^{\circ}-114$; ADBR, B 286; LFM, n० 794 et $\mathrm{n}^{\circ} 899 ; \mathrm{CP}, \mathrm{n}^{\circ} 178 ; \mathrm{LFM}$, $\mathrm{n}^{\circ} 894$; AEA, $\mathrm{n}^{\circ} 91, \mathrm{n}^{\circ} 90^{\circ}$; ADBR, B 287; DelAVILLE LE RoulX, $\mathrm{n}^{\circ} 502$; ACA; Alfons $\mathrm{I}^{\circ}, \mathrm{n}^{\circ} 214$; CSV, $\mathrm{n}^{\circ} 1110, \mathrm{n}^{\circ} 759$; ADBR, $1 \mathrm{H}, \mathrm{n}^{\circ}$ 445, Chartrier (29 mai 1179); Bouche, t. II, ADBR, B 2, fo 41; DelAVILLE, $n^{\circ} 615$; ACA, Alfons $I^{\circ}, n^{\circ} 364$; ADBR, B 292.

63. ADBR, 3 H 6 (1165); teneas et possideas (...) ad forum et consuetudinem Catalonie. Hoc vidilicet modo, ut de predicto castro pacem et guerram per me et per meos facias, tu et tui, et potestatem inde dones michi et meis, iratus et paccatus, quotiescumque eam tibi aut tuis demandaverimus. Salvo preterea semper jure meo meorumque servicio et fidelitate, ADBR, B 293 (décembre 1189); cf. BONNASSIE, p. 765.

64. ADBR, B 287, B 1037, fo $134 \mathrm{v}^{\bullet}$ (janvier 1177); Archives municipales de Marseille, AA 2, $n^{\circ} 3$; AEA, $n^{\circ} 103$; ACA, Alfons $I^{\circ}, n^{\circ} 364$; ADBR, B 38, fo 123 ; $A E A, n^{\circ} 111$ (janvier 1184).

65. ADBR, B 293, B 294, B 297 (février 1195). 
sa campagne militaire en Camargue; à ce titre, il est témoin de la donation du Chaffaut à Rodrico de Cascante. Avec lui, il assista à l'inféodation que le roi fit à Sanche du comté de Rodez, du Carladais et du Gévaudan. Nous le retrouvons, pour la dernière fois, à Aix, dans la cour du roi, parmi les témoins d'un diplôme de mars 1185 confirmant les biens du monastère de Saint-Honorat de Lérins ${ }^{68}$.

C'est à la fin du XIre siècle que la famille de Villeneuve s'est installée dans le comté. Geral de Vilanova, père de Romeu, le baile de Raimond Bérenger $\mathrm{V}$ chanté par Dante et par le folklore provençale7, apparaît pour la première fois en Provence dans la cour aixoise de Raimond Bérenger IV I'année 1178. De retour en Catalogne, il était l'exécuteur testamentaire du vicomte Ramon de la Guardia le 18 octobre 1187. En mai 1193, nous le retrouvons à Marseille. Il allait rester définitivement en Provence; Alphonse II lui concédait en fief les Arcs, Trans, la Motte et les Esclans en 1200 : la même année, Geral était nommé baile d'Antibes par le comte. Il devait mourir sur le sol provençal vers $1225^{\circ 8}$.

La famille de Vilanova était possessionnée dans le Bas Llobregat, au sud de Barcelone, comme en témoigne le testament de Pons de Vilanova en novembre $1179^{\circ 9}$. Pour tout legs, il remettait à son neveu Geral une créance de huit morabatins. A la lecture du testament, l'on s'aperçoit de la faible étendue du domaine dans la région de Sant Boi, où il fait l'objet de divisions successorales, et surtout de la situation assez précaire de Geral de Vilanova qui a dû s'endetter. En Provence, où il apportait son aide aux comtes, sa situation économique et son statut social devaient s'améliorer.

Nous pouvons ainsi conclure sur le caractère catalano-aragonais des cadres politiques de Provence à la fin du $\mathrm{XII}^{\mathrm{e}}$ siècle $^{70}$. Le roi avait besoin d'hommes sûrs et fidèles pour la mise en place de nouvelles structures administratives entre Rhône et Durance et il les cherchait

66. AEA, $\mathrm{n}^{\circ} 90, \mathrm{n}^{\circ} 103$; ACA, Alfons $\mathrm{I}^{\circ}, \mathrm{n}^{\circ} 364 ; \mathrm{CL}, \mathrm{n}^{\circ} 10$.

67. La Divine Comédie, Le Paradis, chant VI; cf., entre autres, Almanach historique, littéraire et biographique de la Provence (Marseille, 1856-1876), page de
couverture.

68. BENoît, op. cit., $\mathrm{n}^{\circ} 10$ (1.er nctobre 1203) ; BUSQUET (R.), op. cit., p. 498 ; AA 2, $\mathrm{n}^{\circ} 3$; ADBR, B 1065, fo $183 \mathrm{v}^{\circ} ; \mathrm{CSC}, \mathrm{n}^{\circ} 1172$.

69. ACA, Alfons $\mathrm{I}^{\circ}, \mathrm{n}^{\circ} 274$ (novembre 1179).

70. De même qu'en Provence, de 1112 à 1250 , * les bailes qui résidaient à Milhau et travaillaient à affermir en Gévaudan la puissance de leur maître, les lieutenants qu'ils désignaient dans les villes secondaires devaient être choisis parmi les Aragonais * (MATHOREZ, Notes sur la pénétration des Espagnols en France du XII' au XIII* siècle, dans Bulletin Hispanique, 1922, p. 42). 
principalement dans la Péninsule Ibérique ${ }^{71}$. De leur côté, quelques cadets de familles nobles trouvaient dans le comté un moyen d'éviter le déclassement ${ }^{72}$. Le comte les casait bien volontiers en Provence Orientale, où ils apportaient une aide précieuse à l'instauration de son pouvoir. Cela fut pour eux le point de départ d'une irrésistible ascension. Leurs descendants continuèrent à fournir du personnel politique aux comtes de la dynastie barcelonaise, bien après la séparation de la Provence du royaume d'Aragon.

Il est difficile de mesurer l'étendue de cette émigration d'élite vers la Provence. Sous le règne d'Alphonse $\mathrm{I}^{\mathrm{er}}, \mathrm{C}$. Delebecque avait constaté l'arrivée à Tarascon d'un certain nombre de familles nobles - les Almoraví, les Auzaran, les Cadafalc - , qu'elle considérait d'origine catalane $^{73}$. Des marchands catalans résidaient en outre à Marseille, comme ce Joan de Conches et ce Guillem de Vilanova que le vicomte Barral prenait, en 1189 , sous sa protection ${ }^{74}$. Quoi qu'il en soit, ce courant migratoire rend compte de l'extraordinaire dynamisme des sociétés catalane et aragonaise de l'époque. Une démographie pléthorique et une grande mobilité sociale expliquent le succès sur les deux fronts musulman et occitan. Elle est le prélude de la grande expansion méditerranéenne du royaume d'Aragon dans les siècles à venir.

71. Contrairement à ce que pensait le grand Vicens Vives (J.) qui croyait à un système de délégation de pouvoirs des comtes de Barcelone aux autochtones des régions qu'ils gouvernaient dans le Midi de la France, à mettre en relation avec le " pactisme" du peuple catalan (Noticia de Catalunya, Barcelone, 1969, $4^{\circ}$ éd., pp. 128-129).

72. RenOUARD (Y.), Les principaux aspects économiques et sociaux de l'histoire des pays de la Couronne d'Aragon aux XIr ${ }^{e}$, XIIr et XIV siecles, dans VII' Congreso de Historia de la Corona de Aragón, 1962, t. III, p. 238.

73. DELEBECOUE (C.), Histoire de la ville de Tarascon depuis les origines jusqu'd l'avènement de la Reine Jeanne (1345), thèse de l'Ecole des Chartes, 1929. Manuscrit déposé aux Archives de Marseille, 24 ii 18-20.

74. ACA, Alfons $I^{\circ}, n^{\circ} 576$ (mars 1189-1190). 